\title{
Bildgebung in der Schwangerschaft
}

Reinhard Loose, Michael Wucherer

\author{
Es gibt immer wieder klinische Situationen, in denen die radiologische \\ Bildgebung während der Schwangerschaft unumgänglich ist. Typische Beispiele \\ sind internistische oder chirurgische Notfälle, insbesondere Unfälle. Bei einer \\ streng gestellten, rechtfertigenden Indikation unter Einbeziehung von Ultraschall \\ und MRT als Alternativen ist bei den meisten Untersuchungen das Risiko der \\ Strahlenanwendung gegenüber dem Nutzen zu vernachlässigen.
}

\section{Einleitung}

Die Bildgebung an schwangeren Patientinnen mit ionisierender Strahlung ist ein sensibles Thema, das allen Beteiligten eine große Sorgfaltspflicht auferlegt. Dies betrifft nicht nur eine geplante Bildgebung bei bekannter Schwangerschaft, sondern auch den Umgang mit Patientinnen, bei denen eine Schwangerschaft zum Untersuchungszeitpunkt nicht bekannt war. Dieser Beitrag beschäftigt sich nicht mit Therapieverfahren in Nuklearmedizin und Strahlentherapie und nicht mit dem beruflichen Strahlenschutz schwangerer Mitarbeiterinnen.

Der medizinische Strahlenschutz beruht auf den 3 Säulen der Rechtfertigung, Optimierung und Dosisbegrenzung. Der eine Untersuchung anfordernde Arzt, der fachkundige Arzt, der die rechtfertigende Indikation stellt, und die/ der durchführende MTRA müssen bei Patientinnen zwischen dem 12. und 55. Lebensjahr immer mit der Möglichkeit einer bestehenden Schwangerschaft rechnen. Die hier genannten Altersgrenzen können sich in unserer multikulturellen Gesellschaft je nach ethnischer Zugehörigkeit leicht nach oben oder unten verschieben.

Merke

Der medizinische Strahlenschutz beruht auf 3 Säulen: Rechtfertigung, Optimierung und Dosisbegrenzung.

\section{Gesetzliche Rahmenbedingungen}

Die Röntgenverordnung (RöV) geht an mehreren Stellen explizit auf Röntgenuntersuchungen an Schwangeren ein [1]:

RöV §23 (3) „Vor einer Anwendung von Röntgenstrahlung in der Heilkunde oder Zahnheilkunde hat der anwendende Arzt gebärfähige Frauen, erforderlichenfalls in Zusammenarbeit mit dem überweisenden Arzt, zu befragen, ob eine Schwangerschaft besteht oder bestehen könnte. Bei bestehender oder nicht auszuschließender Schwangerschaft ist die Dringlichkeit der Anwendung besonders zu prüfen. “
Die Vorgaben von § 23 (3) können auf unterschiedliche Weise erfüllt werden. Im ambulanten Bereich (Röntgenpraxen und klinische Ambulanzen) wird eine Patientin durch den anwendenden Arzt befragt, ob sie schwanger ist oder ob eine Schwangerschaft vorliegen könnte. Diese Befragung kann an eine MTRA delegiert werden, wobei die Verantwortung jedoch beim Arzt verbleibt.

Die Befragung ist zu dokumentieren, falls möglich ist es empfehlenswert, sie sich auch von der Patientin durch Unterschrift bestätigen zu lassen. Im klinischen Alltag kommen in der Regel die wenigsten Patienten direkt in die Radiologie, sondern werden durch Stationen oder Ambulanzen zugewiesen. Hierbei wird in den meisten Fällen bereits auf dem Anforderungsschein zur Röntgenuntersuchung die Frage einer Schwangerschaft gestellt und beantwortet. Es ist dennoch empfehlenswert, dass sich die MTRA die korrekte Antwort auf dem Anforderungsschein noch einmal bestätigen lässt. Bei elektronischen Anforderungen von Röntgenuntersuchungen mit einem sogenannten Order-Entry-System ist in vielen Fällen die Befragung nach einer Schwangerschaft ein Pflichtfeld, ohne dessen Beantwortung eine Anforderung von der Station nicht abgeschickt werden kann. Bei stationären Patientinnen, außer bei Anforderungen durch die Notaufnahme, liegt in den meisten Fällen als verlässlichstes Kriterium bereits der Laborwert eines Schwangerschaftstests vor. Vorsicht ist in jedem Falle bei Patientinnen geboten, die nur eingeschränkt kommunikationsfähig sind oder aus einem anderen Kulturkreis kommen und der deutschen Sprache nur eingeschränkt mächtig sind.

RöV §25 (2) „Ist bei Frauen trotz bestehender oder nicht auszuschließender Schwangerschaft die Anwendung von Röntgenstrahlung geboten, sind alle Möglichkeiten zur Herabsetzung der Strahlenexposition der Schwangeren und insbesondere des ungeborenen Kindes auszuschöpfen.“ 
Die Vorgaben in § 25 (2) sind die wesentlichsten Punkte, mit denen sich dieser Artikel beschäftigt. Ergibt sich während einer bestehenden oder vermuteten Schwangerschaft die Notwendigkeit einer Untersuchung mit bildgebenden Verfahren, ist zunächst zu prüfen, ob die Fragestellung ohne ionisierender Strahlung, z. B. mit Ultraschall oder MRT, zu beantworten ist. Der Aspekt der Vermeidung einer Röntgenuntersuchung ist jedoch nicht der einzige Aspekt bei der Rechtfertigung. Es gibt eine Reihe klinischer Fragestellungen, bei denen eine Bildgebung ohne Röntgenstrahlung keine oder keine hinreichende Aussage liefert. Beispiele hierfür sind Röntgenaufnahmen bei Frakturen des peripheren Skeletts oder die Mammografie bei unklarem Ultraschallbefund und der Kontraindikation gegen eine MRT-Untersuchung mit Kontrastmittel. Es ist daher die besondere Verantwortung bei der Stellung der rechtfertigenden Indikation zu prüfen, ob:

- auf eine Röntgenuntersuchung verzichtet werden kann

- die Untersuchung auf einen späteren Zeitpunkt der Schwangerschaft verschoben werden kann

- ein Röntgenverfahren zwingend eine Kontrastmittelgabe erfordert

- sich alternative Verfahren ohne ionisierender Strahlung anbieten

Hierzu ist es notwendig zu wissen, in welchem Zeitfenster der Schwangerschaft sich eine Patientin befindet. Weiterhin sind elementare Kenntnisse der Strahlenbiologie während einer Schwangerschaft notwendig.

Ist die Indikation zu einer Untersuchung gestellt, sind alle erforderlichen Strahlenschutzmaßnahmen für die werdende Mutter und insbesondere für das ungeborene Kind zu ergreifen. Hierzu gehört neben der Anwendung von Strahlenschutzmitteln (Abschirmung) auch die Optimierung der Untersuchungsparameter, die sich insbesondere im Bereich des Abdomens von nicht schwangeren Patientinnen unterscheiden können.

Anhand vieler praktischer Erfahrungen ist bekannt, dass in der Röntgendiagnostik und in der diagnostischen Anwendung von Radionukliden in deutlich über $90 \%$ der Fälle ein Risiko für eine Schädigung des Embryos bzw. Feten im Sinne einer Fehlbildung nicht vorliegt, da die mit der Untersuchung verbundenen Strahlendosen zu gering sind [2].

RöV §28d (1) An schwangeren Frauen darf Röntgenstrahlung in der medizinischen Forschung nicht angewendet werden. Das Gleiche gilt für Personen, die auf gerichtliche oder behördliche Anordnung verwahrt werden.

Die Einschränkung von § 28d (1) spielt im klinischen Alltag keine Rolle und definiert lediglich, welche Personengruppen nicht in Forschungsprojekte eingeschlossen werden dürfen, bei denen ionisierende Strahlung zur Anwendung kommt.

Merke

Die gesetzlichen Rahmenbedingungen für

Röntgenuntersuchungen an Schwangeren sind in der Röntgenverordnung (RöV) festgelegt.

\section{Grundlagen der Strahlenbiologie in der Schwangerschaft}

Es wird zwischen 2 Kategorien biologischer Strahlenwirkungen unterschieden: deterministische und stochastische Strahlenwirkungen [3]:

Deterministische Wirkungen entstehen durch die Abtötung von Gewebezellen und führen zu einem Ungleichgewicht zwischen Zellnachschub und Zellverlust. Übersteigt dieses Ungleichgewicht eine kritische Schwelle, wird das betroffene Organ oder Gewebe geschädigt. Für deterministische Wirkungen werden Schwellendosen angenommen, unterhalb derer die Anzahl abgetöteter Zellen zu gering ist, um die Funktion von Organen oder Geweben nachhaltig zu beeinträchtigen.

Stochastische Wirkungen entstehen durch Veränderungen der genetischen Information der Zellen (DNS). In der Folge kann es zur Entstehung einer bösartigen Erkrankung wie Krebs oder Leukämie kommen. Dabei liegt zwischen der Bestrahlung und dem Auftreten der Krebserkrankung oder der Leukämie eine so genannt Latenzzeit, die mehrere Jahre oder Jahrzehnte betragen kann. Die Wahrscheinlichkeit, dass stochastische Effekte eintreten, steigt mit zunehmender Dosis. Ein Dosisschwellenwert wie bei den deterministischen Wirkungen wird hier nicht angenommen.

\section{Merke}

Es gibt deterministische und stochastische Strahlenwirkungen.

\section{Auswirkungen}

\section{Deterministische Wirkungen}

Fehlbildungen und Entwicklungsstörungen des Ungeborenen zählen zu den deterministischen Wirkungen einer Strahlenexposition. Ihr Auftreten hängt nicht nur von der Höhe der Strahlendosis ab, sondern auch vom Zeitpunkt der Bildgebung im Schwangerschaftsverlauf [3]:

Die ersten 10 Tage einer Schwangerschaft: Eine Strahlenexposition kann dazu führen, dass sich die befruchtete Eizelle nicht einnistet oder abstirbt. Der Dosisschwellenwert für diesen Effekt liegt bei ca. 100 Millisievert (mSv) (Uterusdosis). 
10. Tag bis 8. Woche: Während der Organogenese teilen und differenzieren sich die Zellen. In dieser Phase besteht das Risiko für Fehlbildungen. In Tierversuchen wurden hierfür Dosisschwellen beobachtet. Für den Menschen werden als Dosisschwellenwert 100 mSv angenommen.

Ab der 10. Schwangerschaftswoche können Strahlenexpositionen eine Fehlentwicklung des Gehirns mit geistiger Retardierung zur Folge haben. Für diese Strahlenwirkung wird eine Schwellendosis von ca. 300 mSv angenommen.

Bezogen auf eine einzelne Untersuchung wird im Rahmen der üblichen radiologischen und nuklearmedizinischen Diagnostik die Schwellendosis von 100 mSv für das Ungeborene im Allgemeinen nicht erreicht.

\begin{abstract}
Merke
Zu den deterministischen Wirkungen einer Strahlenexposition zählen Fehlbildungen und Entwicklungsstörungen des Ungeborenen.
\end{abstract}

\section{Stochastische Wirkungen}

Die Wahrscheinlichkeit für das Eintreten eines stochastischen Spätschadens ist unabhängig von der Entwicklungsphase des Ungeborenen. Es gilt als gesichert, dass das Krebsrisiko, insbesondere das Leukämierisiko, für ein Kind nach einer Strahlenexposition im Mutterleib erhöht ist. In der Oxford-Studie, eine große retrospektive Studie, bei der Kinder, die in utero wegen einer Röntgenuntersuchung der Mutter (überwiegend Pelvimetrie) strahlenexponiert wurden, wird belegt, dass bereits in einem Dosisbereich von 10-50 mSv eine signifikante Erhöhung des Leukämie- und Krebsrate in den ersten 10 Lebensjahren auftritt. Diese Erkenntnis stützt heute sehr die Annahme, dass stochastische Wirkungen linear und ohne Schwellenwerte mit der Dosis zunehmen. Eine pränatale Strahlenexposition von 30 mSv kann die Tumorhäufigkeit im Kindesalter verdoppeln [4].

\section{Merke}

Die Wahrscheinlichkeit für das Eintreten eines stochastischen Spätschadens ist unabhängig von der Entwicklungsphase des Ungeborenen.

Die $>$ Abb. 1 zeigt im zeitlichen Verlauf die einzelnen Phasen einer Schwangerschaft und die möglichen Folgen einer Strahlenexposition in den einzelnen Phasen. Alle Schädigungsarten können auch durch andere Noxen verursacht werden. Die Ereigniswahrscheinlichkeit kann durch genetische und andere individuelle Faktoren beeinflusst werden. Findet eine Röntgenuntersuchung in der Präimplantationsphase einer Schwangerschaft statt, gilt die „alles oder nichts“ Regel, d. h., im Falle einer Zellschädigung kommt es zu einem Absterben des Embryos. Der Schwellenwert liegt bei ca. 100 mSv. In der Phase der Organogenese kann es in Abhängigkeit der Dosis zu körperlichen Fehlbildungen kommen. In der fetalen Phase ab der 8. Woche kann es in Abhängigkeit der Dosis zu geistigen Retardierungen kommen. Das Risiko hierfür wird allerdings mit fortschreitender Schwangerschaftsdauer geringer. Das stochastische Risiko einer malignen Erkrankung (Krebs oder Leukämie) liegt mit 0,006 \% / mSv unter den zuvor beschriebenen deterministischen Risiken, hat allerdings auch keinen Schwellenwert, sodass auch bei Dosen unterhalb 100 mSv ein - wenn auch geringes - Risiko besteht. Das Risiko vererbbarer Defekte ist nochmals um mehr als eine Zehnerpotenz geringer und praktisch kaum quantifizierbar. Die Risiken, Schwellendosen und zeitlichen Phasen einer Schwangerschaft sind in - Tab. 1 dargestellt.

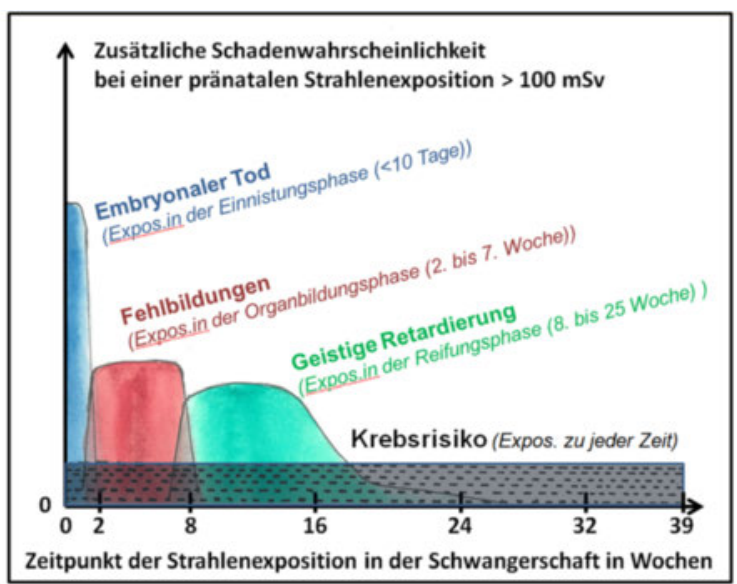

- Abb. 1 Zusätzliche Schadenswahrscheinlichkeit bei hoher pränataler Strahlenexposition des Kindes oberhalb aller Schwellendosen. Für Strahlendosen kleiner 50 mSv kann davon ausgegangen werden, dass ausschließlich das zusätzliche Krebsrisiko entsprechend der Embryodosis besteht und alle anderen Risiken nicht bestehen.

\section{Vorgehen nach Röntgenuntersuchun- gen, wenn eine Schwangerschaft zum Untersuchungszeitpunkt nicht bekannt war}

Nach einer Röntgenuntersuchung mit bis dahin unbekannter Schwangerschaft gilt es zunächst die werdende Mutter zu beruhigen, da bei den allermeisten Untersuchungen die Dosis am Uterus bzw. die Dosis des Ungeborenen sehr gering ist und damit nur ein minimales Risiko besteht. Man kann Röntgenuntersuchungen grob in 3 Gruppen einteilen:

- Am niedrigsten ist die Uterusdosis bei Röntgenaufnahmen des peripheren Skeletts und des Schädels, vorausgesetzt, die notwendigen Strahlenschutzmittel werden verwendet.

- Die nächste Stufe betrifft Röntgenuntersuchungen des Körperstamms, ohne dass der Uterus im direkten Strahlengang liegt.

- Die 3. Stufe bezieht sich auf Röntgenuntersuchungen, bei denen der Uterus direkt exponiert wird. 
> Tab. 1 Effekte, Zeitraum nach Konzeption, Schwellendosis und Risiko-Koeffizienten einer Strahlenexposition während der Schwangerschaft [2].

\begin{tabular}{|c|c|c|c|}
\hline Effekt & $\begin{array}{l}\text { Zeitraum nach } \\
\text { Konzeption }\end{array}$ & $\begin{array}{l}\text { Unterer Schwellenwert der } \\
\text { Dosis für Auftreten des Effekts }\end{array}$ & Risikokoeffizient \\
\hline $\begin{array}{l}\text { Tod während der } \\
\text { Präimplantationsphase }\end{array}$ & -10 Tage & $100 \mathrm{mSv}$ & $0,1 \%$ pro $\mathrm{mSv} *$ \\
\hline Missbildungen & 10 Tage bis 8 . Woche & $100 \mathrm{mSv}$ & $0,05 \%$ pro $\mathrm{mSv} *$ \\
\hline $\begin{array}{l}\text { Schwere geistige } \\
\text { Retardierung }\end{array}$ & $\begin{array}{l}\text { - 8.-15. Woche } \\
\text { - 16.-25. Woche }\end{array}$ & $\begin{array}{l}\text { - } 300 \mathrm{mSv} \\
\text { - } 300 \mathrm{mSv}\end{array}$ & $\begin{array}{l}\text { - } 0,04 \% \text { pro mSv* } \\
\text { - } 0,01 \% \text { pro mSv* }\end{array}$ \\
\hline IQ-Reduktion (Intelligenztest) & $\begin{array}{l}\text { - 8.-15. Woche } \\
\text { - 16.-25. Woche }\end{array}$ & & $\begin{array}{l}\cdot \text { - 0,03 IQ pro mSv } \\
\cdot 0,01 \mathrm{IQ} \text { pro } \mathrm{mSv}\end{array}$ \\
\hline maligne Erkrankungen & & & $0,006 \%$ pro mSv \\
\hline vererbbare Defekte & & & $\begin{array}{l}\text { - } 0,0003 \% \text { pro mSv männlich } \\
\text { - } 0,0001 \% \text { pro mSv weiblich }\end{array}$ \\
\hline
\end{tabular}

Die > Abb. 2 stellt die Dosis typischer Untersuchungen dar.

In Abhängigkeit der Dosis des Uterus wird im Weiteren ein Vorgehen in 3 Stufen empfohlen:

- Stufe1: grobe Abschätzung nach Tabellen (bis max. $20 \mathrm{mSv}$ )

- Stufe 2: Abschätzung durch Berechnung mit Untersuchungsparametern und geräte- und patientenspezifischen Daten (20-100 mSv)

- Stufe 3: Berechnung auf der Basis individueller Untersuchungsparameter und geräte- und patientenspezifischen Daten (Dosis > 100 mSv); es wird empfohlen, einen Medizinphysiker hinzuzuziehen.

Ein Entscheidungsbaum zum weiteren Vorgehen ist in - Abb. 3 dargestellt, er ist im Vergleich zur Empfehlung der DGMP leicht verändert [2]. Hier wird auch bei hohen Dosen die Möglichkeit eines Schwangerschaftsabbruchs einbezogen.

\section{Vorgehen bei geplanter \\ Röntgenuntersuchung während einer Schwangerschaft}

Bei bekannter Schwangerschaft ist vor einer Untersuchung zunächst zu prüfen, ob eine bildgebende Diagnostik notwendig ist und ob sie ggf. mit Verfahren ohne ionisierende Strahlung, wie Ultraschall oder MRT, durchgeführt werden kann. Ist nach sorgfältiger Nutzen-Risiko-Abwägung die Indikation zu einer Röntgenuntersuchung gestellt, müssen alle technischen Mittel zum Strahlenschutz und zur Dosisreduktion angewendet werden. Untersuchungen, bei denen sich der Uterus nicht im direkten Strahlengang befindet, sind hierbei in der Regel unkritisch. Aufgrund des besonderen Risikos in der Schwangerschaftsphase der Organogenese sollten Röntgenuntersuchungen, bei denen

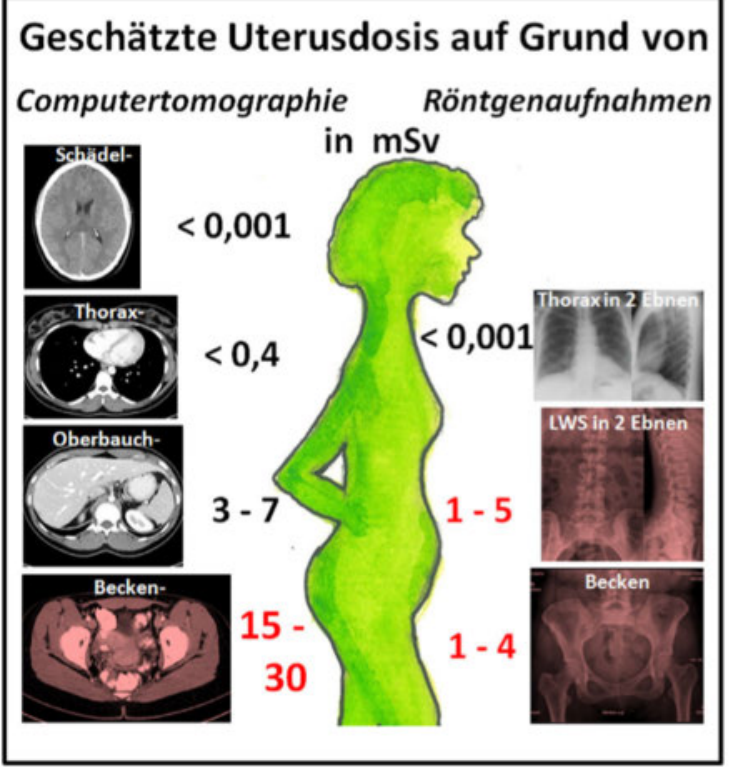

- Abb. 2 Uterusdosen bei CT und konventionellen Röntgenaufnahmen in mSv. Die Ganzkörperdosis des ungeborenen Kindes wird für die ersten 8 Wochen einer Schwangerschaft der Gebärmutterdosis gleichgesetzt. Die in rot markierten Bilder und Werte zeigen an, dass der Uterus ganz bzw. zum Teil im Nutzstrahl liegt. Die Dosiswerte variieren sehr stark mit der Schwangerschaftswoche, der Einstelltechnik und der Statur der Patientin.

keine Notfallindikation besteht, möglichst verschoben werden. Eine Schwangere sollte vor der Untersuchung beruhigend - aber sachlich - über die Rechtfertigung und damit die Nutzen-Risiko-Analyse aufgeklärt werden. Es empfiehlt sich, diese Aufklärung schriftlich vom durchführenden Arzt dokumentieren zu lassen. Im Übrigen gelten alle Risikobetrachtungen des vorigen Absatzes bei unbekannter Schwangerschaft. 


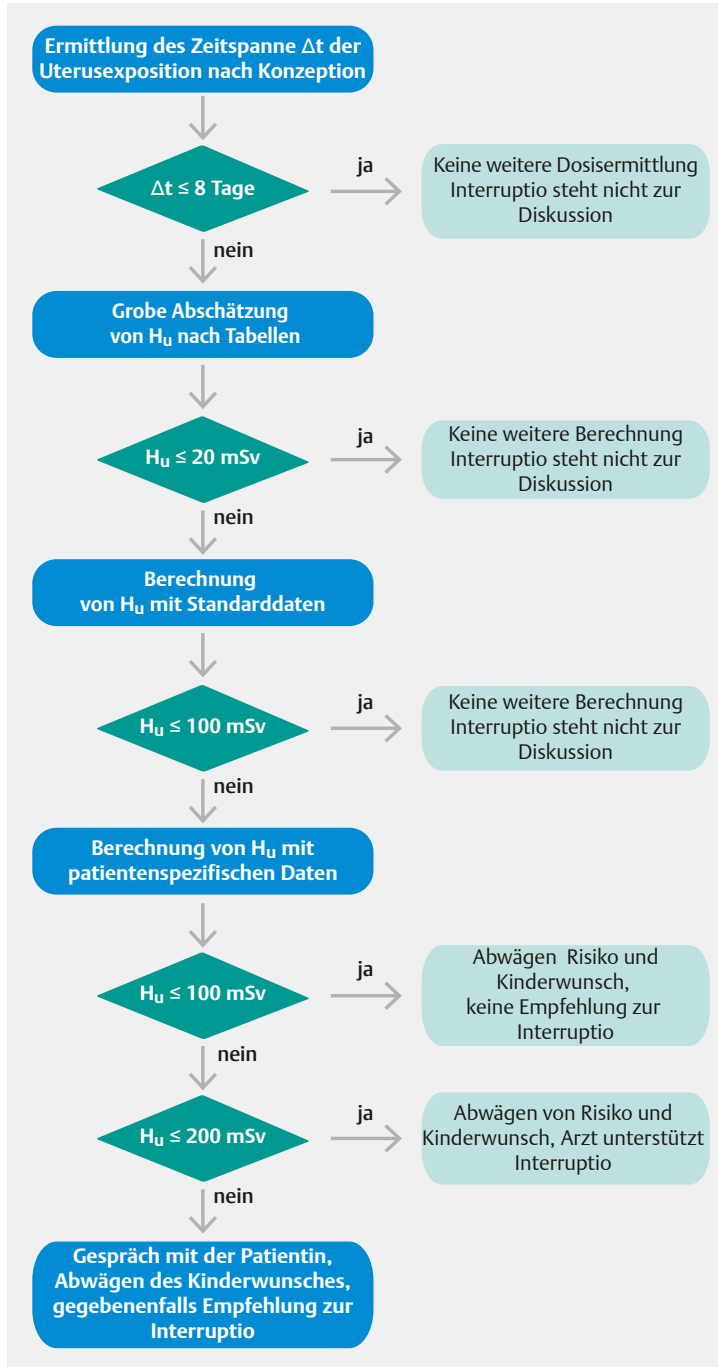

- Abb. 3 Entscheidungsbaum zum Vorgehen nach Exposition einer Schwangeren im Hinblick auf deterministische Schäden beim Kind. $\mathrm{H}_{u}$ ist die ermittelte Dosis des Embryos/Fetus. Ein Gespräch mit der Patientin sollte immer und angemessen erfolgen.
Einen wichtigen Beitrag zum Strahlenschutz liefert bei Röntgenaufnahmen eine gute Kollimation und in der CT eine optimale Begrenzung der Scanlänge nach kaudal. Die A Abb. 4 zeigt Lage und Größe des Uterus und damit auch ungefähr des Kindes während einer fortschreitenden Schwangerschaft. Man kann hierbei gut erkennen, dass z. B. bei lateralen Aufnahmen der LWS bei guter ventraler Einblendung große Teile des Uterus außerhalb des Direktstrahls liegen.

Betrachtet man die Dosis des Uterus bei einer Bildgebung in der Schwangerschaft, kann man die Röntgenuntersuchungen in 3 Gruppen aufteilen (siehe auch Abb. 2).

1. Niedrige Exposition:

- Röntgen der Extremitäten, Kopf, Thorax (außer Hüfte)

- Uterusdosis < 0,1 mGy $\rightarrow$ die Untersuchung ist jederzeit möglich

\section{Mittlere Exposition:}

- Untersuchungen des Körperstamms ohne direkte Exposition des Uterus

- Uterusdosis < 1 mGy $\rightarrow$ die Untersuchung hinterfragen und adaptieren, zum Schutz vor extrafokaler Streustrahlung die Region des Uterus und der Umgebung soweit als möglich abdecken (umschließende Bleischürze)

\section{Höhere Exposition:}

- Untersuchungen des Körperstamms mit Uterus im Strahlungsfeld

- Uterusdosis > 1 mGy (Becken Durchleuchtung 20-100 mGy, Becken-CT bis zu 50 mGy/Serie) $\rightarrow$ die Untersuchung verschieben, außer bei hoher Dringlichkeit, die erforderliche Bildqualität optimieren und das Untersuchungsvolumen sinnvoll begrenzen.

Falls möglich und verfügbar, sollte bei niedrigen Expositionen der Gruppe 1 eine Optimierung der Uterusdosis, ggf. eine Dosimetrie, durchgeführt und ein Medizinphysiker hinzugezogen werden. Als Dosisreduktionsmaßnahme

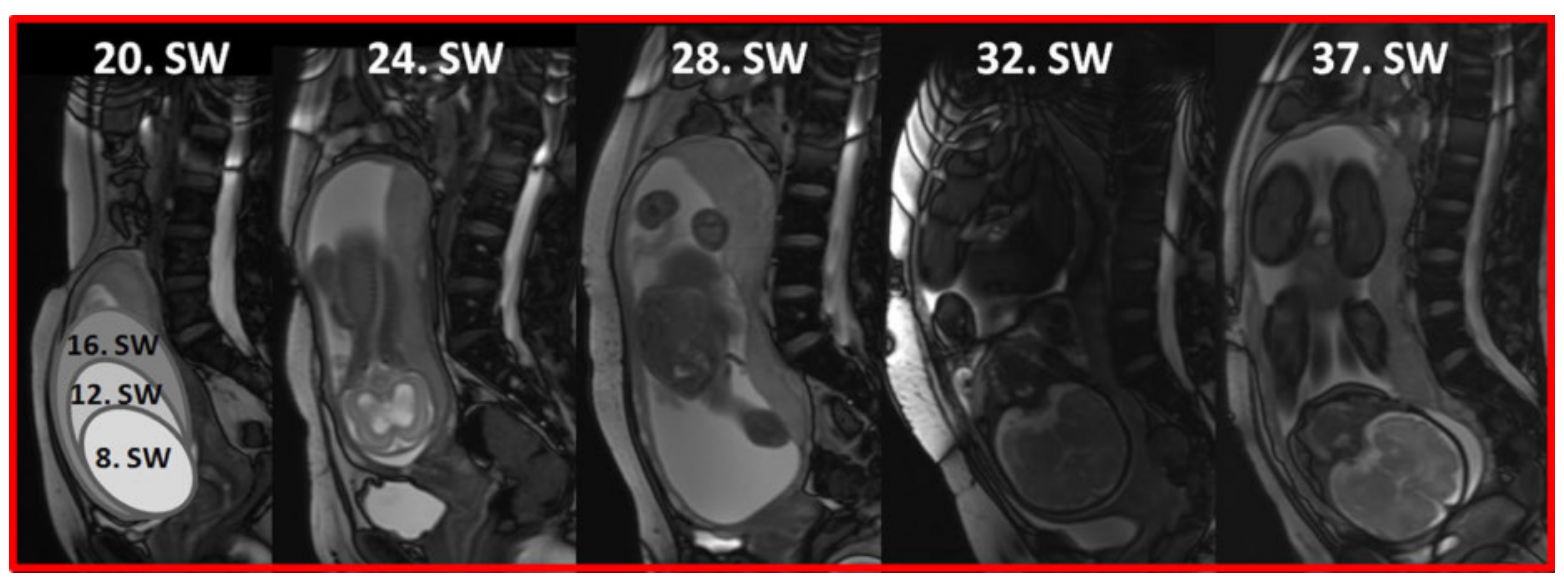

- Abb. 4 Lage und Größe des Uterus im Verlauf der Schwangerschaft in Abhängigkeit der Schwangerschaftswoche (SW). Sagittale MRT-Schnittbilder unterschiedlicher Patientinnen. Bild: Dr. Susanne Schulz-Heise, Abt. für Kinderradiologie, Universitätsklinikum Erlangen. 
neben der optimalen Einstelltechnik sollten mit Bleischürzen der Unterleib so gut wie möglich vor extrafokaler und Streustrahlung geschützt werden. Für die Dosisermittlung kann ein elektronisches Dosimeter auf Uterushöhe positioniert werden. Auch wenn die Reduktionsmaßnahmen sehr gering sein können, ist dieses Verhalten gegenüber der Patientin auch aus psychologischen Gründen empfehlenswert.

Merke

Dosisreduktionsmaßnahmen: eine optimale Einstelltechnik und die Verwendung von Bleischürzen, um den Unterleib so gut wie möglich vor extrafokaler und Streustrahlung zu schützen.

\section{Typische Indikationen und Empfehlun- gen zu Röntgenuntersuchung während einer Schwangerschaft}

Die $>$ Tab. 2 zeigt eine Reihe typischer Symptome bzw. Erkrankungen, die im Verlauf einer Schwangerschaft auftreten können [5]. Während einer Schwangerschaft, insbesondere im fortgeschrittenen Stadium, kommt es zu einer zunehmenden Kompression der Beckenvenen und der V. cava durch den Uterus. Kommt es hierdurch zu einer Lungenembolie mit kardinalen Belastungszeichen, ist eine Bildgebung unumgänglich. Es wurde in der Vergangenheit oft diskutiert, ob zur Abklärung ein Kontrastmittel-CT der Lunge oder eine Ventilations-Perfusions-Szintigrafie besser geeignet ist. Die - Tab. 3 zeigt anhand der Dosisdaten, dass die CT in allen 3 Schwangerschaftsphasen der nuklearmedizinischen Diagnostik überlegen ist. Insbesondere in der Frühschwangerschaft ist der Uterus weit vom Scanvolumen der CT entfernt, während der Embryo sehr nahe an eventuell ausgeschiedener Blasenaktivitäten zu liegen kommt.

\section{Kontrastmittel}

Die intravenöse Gabe jod- oder gadoliniumhaltiger Kontrastmittel bedarf wie die Röntgenuntersuchung selbst ebenfalls einer strengen Indikationsstellung. Fast alle Kontrastmittel haben keine Zulassung zur Anwendung in der Schwangerschaft und enthalten lediglich den Hinweis, dass sie in Tierexperimenten nicht teratogen sind [7]. Nähere Angaben zu den einzelnen Kontrastmitteln finden sich z. B. in der Roten Liste [8].

Jodhaltige Kontrastmittel passieren die Plazenta. Da die fetale Schilddrüse im 2. und 3. Trimenon Jod aktiv aufnimmt und Schilddrüsenhormone produziert, kann die Gabe von jodhaltigem Kontrastmittel während der Schwangerschaft postpartal Schilddrüsenstörungen beim Neugeborenen verursachen; hier sind sowohl Hyperthyreosen als auch Hypothyreosen möglich.

> Tab. 2 Klinische Leitsymptome, für deren Abklärung Röntgenuntersuchungen auch bei Schwangeren als primäres oder sekundäres Verfahren eingesetzt werden (modifiziert nach [5])

\begin{tabular}{|c|c|c|}
\hline Leitsymptom & mögliche Ursachen & $\begin{array}{l}\text { primäres Untersuchungsverfahren } \\
\text { (sekundäres Verfahren) }\end{array}$ \\
\hline Dyspnoe & $\begin{array}{l}\text { - Lungenembolie } \\
\text { - Pneumonie, Pneumothorax }\end{array}$ & $\begin{array}{l}\text { - Venenduplex (CT-Angiografie) } \\
\text { - Röntgen Thorax }\end{array}$ \\
\hline $\begin{array}{l}\text { akutes/unklares } \\
\text { Abdomen }\end{array}$ & $\begin{array}{l}\text { - Ileus } \\
\text { - Uroltihiasis }\end{array}$ & $\begin{array}{l}\text { - Ultraschall (CT) } \\
\text { - Ultraschall (CT Low-Dose) }\end{array}$ \\
\hline Trauma & $\begin{array}{l}\text { - Polytrauma, Wirbelsäulenfraktur, Schädel-Hirn-Trauma, } \\
\text { Bauchtrauma } \\
\text { - Extremitätenfraktur }\end{array}$ & $\begin{array}{l}\text { - } \mathrm{CT} \\
\text { - Röntgen konventionell }\end{array}$ \\
\hline $\begin{array}{l}\text { Akuter } \\
\text { Kopfschmerz }\end{array}$ & $\begin{array}{l}\text { - Verdacht auf intrazerebrale Blutung/Subarachnoidalblutung } \\
\text { - Verdacht auf Sinusvenenthrombose }\end{array}$ & $\begin{array}{l}\text { - CT kraniell } \\
\text { - CT kraniell }\end{array}$ \\
\hline Apoplex & & - CT-Angiografie \\
\hline
\end{tabular}

- Tab. 3 Fetale Dosis der Ventilations-/Perfusionsszintigrafie mit 99mTc, der pulmonalen CT-Angiografie und Dosisquotient beider Verfahren. Bei Verzicht auf die Ventilation halbiert sich die Dosis etwa, liegt aber immer noch deutlich über der CT-Angiografie, Tabelle modifiziert nach [6].

\begin{tabular}{|l|c|c|}
\hline Uterusdosis & Frühschwangerschaft & Mittlere Schwangerschaft \\
\hline $\begin{array}{l}\text { der Ventilations und Perfusionsszintigrafie } \\
{[\mu G y]}\end{array}$ & 740 & 680 \\
\hline der pulmonalen CT-Angiografie $[\mu \mathrm{Gy}]$ & $3-20$ & $8-80$ \\
\hline Quotient: Szintigrafie/CT & $>40$ & $>10$ \\
\hline
\end{tabular}


Bei gadoliniumhaltigen Kontrastmitteln ist nicht nur das Risiko einer nephrogenen systemischen Fibrose bekannt, es wurden jetzt auch Fälle von Gadoliniumablagerungen im Gehirn beschrieben, die in ihrer klinischen Bedeutung noch nicht einzuordnen sind.

Aktuelle Hinweise und Empfehlungen zur Anwendung von Kontrastmitteln in der Schwangerschaft finden sich bei der European Society of Urogenital Radiology [9].

Merke

Die intravenöse Gabe jod- oder gadoliniumhaltiger Kontrastmittel bedarf einer strengen Indikationsstellung.

\section{KERNAUSSAGEN}

- Die Bildgebung an schwangeren Patientinnen mit ionisierender Strahlung ist ein sensibles Thema.

- Bei bekannter Schwangerschaft ist vor einer Untersuchung zunächst zu prüfen, ob eine bildgebende Diagnostik notwendig ist und ob sie ggf. mit Verfahren ohne ionisierende Strahlung, wie Ultraschall oder MRT, durchgeführt werden kann.

- Der medizinische Strahlenschutz beruht auf den drei Säulen der Rechtfertigung, Optimierung und Dosisbegrenzung.

- Radiografische Aufnahmen, bei denen der Uterus nicht Zielvolumen liegt, können als unkritisch angesehen werden.

- In allen Fällen ist eine beruhigende Aufklärung der Schwangeren und die Optimierung der Untersuchung unter Anwendung aller sinnvollen Strahlenschutzmaßnahmen notwendig.

\section{Interessenskonflikt}

Die Autoren geben an, dass keine Interessenkonflikte vorliegen.

Über die Autoren

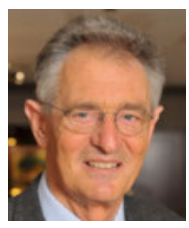

Prof. Dr. med. Dr. rer. nat. Reinhard Loose 1967-1975 Studium der Physik, 1984 Promotion. 1993 Promotion in Medizin und Itd. Oberarzt im Institut für Klinische Radiologie, Universität Mannheim, 1996 Habilitation. Seit 2010 APL Professor in Radiologie der Universität Erlangen-Nürnberg. 1996-2016 Chefarzt am Institut für Diagnostische und Interventionelle Radiologie und Klinik und Institut für Nuklearmedizin, Klinikum Nürnberg.

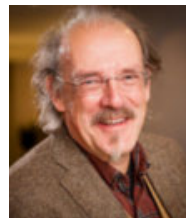

\section{Dr. rer. nat. Michael Wucherer}

1975-1985 Studium der Physik mit anschlieBender Promotion. 1982-1992 Tätigkeit als Medizinphysiker an der Friedrich-AlexanderUniversität Erlangen-Nürnberg und anschlieBend am Institut für Medizinische Physik im Klinikum Nürnberg. Seit 2000 Leiter des Institutes für Medizinische Physik, Leiter der MTRA-Schule und Strahlenschutzbevollmächtigter für das Klinikum Nürnberg. Berater in zahlreichen Strahlenschutzgremien.

\section{Korrespondenzadresse}

\section{Dr. Michael Wucherer}

Klinikum Nürnberg, Institut für Medizinische Physik

Paracelsus Medizinische Privatuniversität

Klinikum Nürnberg Nord

Prof.-Ernst-Nathan-Str. 1

90419 Nürnberg

Deutschland

Tel: +49(0)911/398-3663

Fax: +49(0)911/398-3288

E-Mail: wucherer@klinikum-nuernberg.de

Literatur

[1] Verordnung über den Schutz vor Schäden durch Röntgenstrahlen (Röntgenverordnung - RöV). Im Internet: www. gesetze-im-internet.de/bundesrecht/r_v_1987/gesamt.pdf; Stand: 29.05.2017

[2] Deutsche Gesellschaft für Medizinische Physik e. V. DGMPBericht Nr. 7. Pränatale Strahlenexposition aus medizinischer Indikation. Dosisermittlung, Folgerungen für Arzt und Schwangere. Im Internet: www.dgmp.de/media/document/ 205/bericht7-neuauflage2002.pdf; Stand: 29.05.2017

[3] Bundesamt für Strahlenschutz. Ionisierende Strahlung. Im Internet: http://www.bfs.de/DE/themen/ion/anwendungmedizin/diagnostik/schwangerschaft/schwangerschaft_ node.html; Stand: 29.05.2017

[4] Strahlenschutzkommission. Strahlenschutz für das ungeborene Kind, Empfehlung der Strahlenschutzkommission und Wissenschaftliche Begründung. Im Internet: http://www.ssk.de/ SharedDocs/Beratungsergebnisse_PDF/2004/Strahlenschutz_ ungeborenesKind.pdf?_blob=publicationFile; Stand: 29.05.217

[5] Hojreh A, Prosch H, Karanikas G et al. Schutz des ungeborenen Lebens bei diagnostischen und interventionellen radiologischen Verfahren. Radiologe 2015; 55: 663-672

[6] Groves AM, Yates S], Win T et.al. CT Pulmonary Angiography versus Ventilation-Perfusion Scintigraphy in Pregnancy: Implications from a UK Survey of Doctors' Knowledge of Radiation Exposure. Radiology 2006; 240: 765-770

[7] Weisser G, Steil V, Neff KW et al. Radiologie und Schwangerschaft. Radiologe 2012; 52: 81-92

[8] Rote Liste. Im Internet: http://www.roteliste.de; Stand: 29.05.2017

[9] European Society of Urogenital Radiology. Contrast Media Education. Im Internet: www.esur.org/contrast-mediaeducation/; Stand: 29.05.2017

\section{Bibliographie}

DOI https://doi.org/10.1055/s-0043-103146

Radiopraxis 2017; 10: 131-138

(c) Georg Thieme Verlag KG Stuttgart · New York ISSN 1866-1033 


\section{Punkte sammeln auf CM/E.thieme.de}

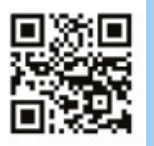

Diese Fortbildungseinheit ist 12 Monate online für die Teilnahme verfügbar. Sollten Sie Fragen zur Online-Teilnahme haben, finden Sie unter http://cme.thieme.de/hilfe eine ausführliche Anleitung. Wir wünschen viel Erfolg beim Beantworten der Fragen!

Unter eref.thieme.de/ZZX8MFK oder über den QR-Code kommen Sie direkt zum Artikel zur Eingabe der Antworten.

\section{Frage 1}

Die rechtfertigende Indikation für eine Röntgenuntersuchung stellt
A die MFA.
B die MTRA.
C der Arzt mit der erforderlichen Fachkunde.
D die Schwangere selbst.
E die MTRA-Schülerin.

\section{Frage 2}

Die Fetogenese erstreckt sich von der
A 1.-2. Woche.
D 8.-39. Woche.
B 2.-8. Woche.
E 4.-20. Woche.
C 4.-16. Woche.

\section{Frage 3}

Die Uterusdosis ist am niedrigsten bei
A Röntgenaufnahmen des peripheren Skeletts.
B Röntgenaufnahmen des Körperstamms.
C Röntgenaufnahmen mit direkter Uterusexposition.
D Abdomen-CT.
E Ist bei allen vier genannten Untersuchungstypen gleich.

\section{Frage 4}

Ab welcher Uterusdosis Hu wird ggf. eine Interruptio erwogen?
A $\mathrm{H}_{\mathrm{u}} \cong 2 \mathrm{mSv}$
D $\mathrm{H}_{\mathrm{u}} \cong 50 \mathrm{mSv}$
B $\mathrm{H}_{\mathrm{u}} \cong 10 \mathrm{mSv}$
E $H_{u}>100 \mathrm{mSv}$
C $\mathrm{H}_{\mathrm{u}} \cong 20 \mathrm{mSv}$

\section{Frage 5}

Bei welchem Symptom ist keine Röntgenuntersuchung während der Schwangerschaft anzufertigen?
A Lungenembolie
B Wirbelsäulenfraktur
C Bauchtrauma
D unkomplizierte Nasennebenhöhlenentzündung
E Verdacht auf Subarachnoidalblutung

\section{Frage 6}

Welche Aussage zur Kontrastmittelgabe während der Schwangerschaft ist falsch?
A i. v.-Gabe von jod- oder gadoliniumhaltiger Kontrastmittel bedarf einer strengen Indikationsstellung.
B Jodhaltige Kontrastmittel passieren die Plazenta.

C Jodhaltige Kontrastmittel passieren nicht die Plazenta.

D Jodhaltige Kontrastmittel können postpartale Schilddrüsenstörungen verursachen.

E Gadoliniumhaltige Kontrastmittel können eine nephrogene systemische Fibrose verursachen.

\section{Frage 7}

Was ist keine Strahlenschutzmaßnahme?
A optimale Begrenzung der Scanlänge nach kaudal in $\operatorname{der} \mathrm{CT}$
B bei Röntgenaufnahmen eine gute Kollimation
C strenge Indikationsstellung
D der Patientin eine Bleischürze anziehen
E den Röntgentisch vor der Untersuchung desinfizieren

\section{Frage 8}

Welches bildgebende Verfahren wird bei Verdacht auf Sinusvenenthrombose empfohlen?
A Thoraxröntgen
B Abdomenröntgen
C Schädelröntgen
D Schädel-CT(CCT)
E Ultraschall

\section{Frage 9}

Welches Schadensrisiko besteht bei pränataler Strahlenexposition unabhängig von Zeitpunkt der Exposition während der Schwangerschaft
A pränatalerTod
B Organmissbildung
$C$ geistige Retardierung
D Leukämie
E Fehlbildungen des Skeletts

\section{Frage 10}

Ein Order-Entry-System ist
A Eine spezielle radiologische Handy-App.
$B$ Ein System für elektronische Anforderungen von Röntgenuntersuchungen.
C Ein spezielles Arzttelefon.
D Ein spezielles Befundungssystem.
E Ein Bilderarchivierungssystem. 